\title{
Effect of toroidal field ripple on the formation of internal transport barriers
}

\author{
P C de Vries ${ }^{1}$, E Joffrin ${ }^{2}$, N C Hawkes ${ }^{1}$, X Litaudon ${ }^{3}$ C D Challis ${ }^{1}$, \\ Y Andrew $^{1}$, M Beurskens ${ }^{1}$, M Brix ${ }^{1}$, J Brzozowski ${ }^{4}$, K Crombé ${ }^{5}$, \\ C Giroud $^{1}$, J Hobirk ${ }^{6}$, T Johnson ${ }^{4}$, J Lönnroth ${ }^{7}$, A Salmi ${ }^{7}$, T Tala ${ }^{8}$, \\ V Yavorskij ${ }^{9}$, K-D Zastrow ${ }^{1}$ and JET EFDA Contributors ${ }^{10}$ \\ JET-EFDA Culham Science Centre, OX14 3DB, Abingdon, UK \\ ${ }^{1}$ EURATOM/UKAEA Fusion Association, Culham Science Centre, Abingdon, OX14 3DB, UK \\ 2 EFDA-JET CSU, Culham Science Centre, Abingdon, Oxfordshire, OX14 3DB, UK \\ ${ }^{3}$ Association EURATOM-CEA, DSM/DFRC, CEA Cadarache, 13108, \\ St Paul lez Durance, France \\ ${ }^{4}$ Association EURATOM-VR, Fusion Plasma Physics, EES, KTH, Stockholm, Sweden \\ ${ }^{5}$ Department of Applied Physics, Ghent University, Ghent, Belgium \\ ${ }^{6}$ Max-Planck-Institut für Plasmaphysik, Euratom Association, 85748 Garching, Germany \\ ${ }^{7}$ Association Euratom-Tekes, Helsinki University of Technology, PO Box 4100, \\ 02015 TKK, Finland \\ ${ }^{8}$ Association Euratom-Tekes, VTT, PO Box 1000, 02044 VTT, Finland \\ ${ }^{9}$ Institute for Theoretical Physics, Association EURATOM-OEAW, \\ University of Innsbruck, Austria \\ E-mail: Peter.de.Vries@jet.uk
}

Received 23 November 2007, in final form 17 January 2008

Published 10 April 2008

Online at stacks.iop.org/PPCF/50/065008

\begin{abstract}
The effect of a toroidal field (TF) ripple on the formation and performance of internal transport barriers (ITBs) has been studied in JET. It was found that the TF ripple had a profound effect on the toroidal plasma rotation. An increased TF ripple up to $\delta=1 \%$ led to a lower rotation and reduced the rotational shear in the region where the ITBs were formed. ITB triggering events were observed in all cases and it is thought that the rotational shear may be less important for this process than, for example, the $q$-profile. However, the increase in the pressure gradient following the ITB trigger was reduced in discharges with a larger TF ripple and consequently a lower rotational shear. This suggests that toroidal rotation and its shear play a role in the growth of the ITB once it has been triggered.
\end{abstract}

10 See the appendix of Watkins M L et al 2006 Proc. 21 st Int Conf. on Fusion Energy 2006 (Chengdu) (Vienna: IAEA). 


\section{Introduction}

Advanced tokamak scenarios are proposed to provide the conditions needed for a steady-state demonstration in ITER [1-5]. The goal is to reach a high pressure at a modest plasma current where a large fraction of the plasma current is driven by the bootstrap current, providing a noninductive self-generated current. These scenarios often involve modification of the current profile, which has been shown to lead to internal transport barriers (ITBs) that improve the confinement and, hence, performance of the fusion plasma [6,7].

The physical mechanism behind ITB formation has not yet been clearly identified. Studies of the physics of ITBs in tokamak plasmas have indicated two factors that play a crucial role in their formation and development: magnetic and rotational shear [6]. It was found, for example, that ITBs form more easily in plasmas that have a specific current density profile and safety factor, $q$, profile, such that there exists a region of negative magnetic shear, $q^{\prime} / q$ [8]. Modelling studies have shown the effect of rotational shear on the ITB in discharges with a small but positive magnetic shear [9]. Both magnetic and rotational shears are believed to play a role in turbulence suppression, creating a region with reduced transport. Other factors are also thought to affect ITBs, for example, turbulence suppression induced by the Shafranov shift [10] or zonal flows [11]. These possible mechanisms do not necessarily act independently and it is possible that ITB formation is an interplay of more than one mechanism.

The initial formation, or triggering, of the ITB is often attributed to the characteristics of the magnetic shear profile in JET, although external triggers such as MHD events also have been found to play a role $[12,13]$. Further development or strengthening of the ITB could be a highly non-linear process, in which various mechanisms modify the current profile and local magnetic shear or enhance the rotational shear resulting in a further suppression of turbulence. Experimentally it was observed that the further strengthening of a transport barrier, and thus increasing the pressure gradient, could enhance the off-axis driven bootstrap current, yielding further reversal of the magnetic shear and even the formation of current holes in the centre of the plasma [14-16].

At JET, advanced tokamak scenarios usually use predominantly neutral beam injection (NBI) as auxiliary heating. The large toroidal torque by the JET NBI system, and consequently fast toroidal rotation, can enhance the rotational shear. However, it has not evident that NBI heating or rotation is a prerequisite for ITB production. Experiments to produce ITBs with low torque have been performed at JET by increasing the fraction of ion cyclotron resonance heating (ICRH). However, the variety of differences between the NBI and ICRH, such as the power deposition profiles, ion and electron heating fraction or current drive characteristics, adds to the difficulty in interpreting the experimental results [17, 18]. In other devices the possibility of balanced or perpendicular NBI allows a decoupling between NBI heating and the torque it supplies. Experiments showed that a reduction in the rotational shear could degrade the ITB $[19,20]$.

Because of the finite number of toroidal field (TF) coils, a periodic toroidal variation (ripple) of the main magnetic field exists in tokamaks. JET has the unique ability to adjust the amplitude of this ripple, and the value of this feature has been demonstrated in previous experiments [21]. It has been shown that the TF ripple can have a profound effect on the plasma rotation [22]. Hence, tuning the TF ripple provides the possibility of altering the plasma rotation and rotational shear in JET without major changes to the scenario, such as the NBI and ICRH heating schemes.

Beside its effect on plasma rotation, the influence of the TF ripple on advanced tokamak scenarios in general is of importance for ITER. The TF ripple of ITER will differ from JET due to a lower number of TF coils, with 18 and 32 coils in these devices, respectively. It is 
proposed that ferrite material will be mounted between the ITER coils in order to reduce the ripple [23]. Nevertheless, the estimated TF ripple in ITER is expected to be significantly larger than in standard JET plasmas. As well as altering the rotation, the TF ripple may also act on the H-mode pedestal and edge localized modes (ELMs), which have been found to degrade ITBs in JET [24]. A series of dedicated experiments were carried out in order to study the influence of the TF ripple and plasma rotation on the formation and performance of ITBs.

Firstly, the method for varying the TF ripple at JET is discussed in section 2. Here the experimental conditions are described and we introduce the tokamak scenarios in which the ITBs were studied. Section 3 deals with the formation, or triggering, of ITBs. It is important to bear in mind that the triggering processes may not be related to the same physics mechanisms responsible for the subsequent continued growth of the barrier. In section 4 the effect of the TF ripple and plasma rotation on the strength of the ITB, and hence the performance of the scenario, is shown. Finally the results will be summarized and discussed in section 5 .

\section{TF ripple experiments at JET}

Standard operations at JET are carried out with a set of 32 TF coils all carrying equal current. At JET it is possible to vary the TF ripple amplitude by independently powering the 16 odd and 16 even numbered coils [21]. The imbalance current between the two coils set can be changed in a controlled way increasing the TF ripple up to $\delta \sim 3 \%$. Here the TF ripple amplitude, $\delta$, is defined as the relative amplitude of the magnetic field variation: $\delta=\left(B_{\max }-B_{\min }\right) /\left(B_{\max }+B_{\min }\right)$. The estimated maximum TF ripple at the separatrix in ITER (with ferrite material) may be in the order of $\delta \sim 0.5 \%$ (at the full field) [23] while this value in JET is only $\delta \sim 0.08 \%$. The maximum ripple at the separatrix is typically found at the outboard mid-plane.

The TF ripple breaks the axi-symmetry of the magnetic field and enhances particle losses, in particular, energetic ions such as alpha particles created in fusion reactions and those injected by NB or accelerated by ICRH. These non-ambipolar ion losses can have a non-negligible effect on the plasma rotation. Particle losses also result in a reduction in the absorbed power compared with the total power injected by the NBI system. In order to be able to compare discharges with various TF ripple amplitudes in these experiments, the actual absorbed auxiliary heating powers must be determined. The ripple induced power loss fraction and the total absorbed power for these experiments have been modelled using the JAEA Orbit Following Monte Carlo (OFMC) code [25]. Shine-through losses were not included in these calculations, but are largely independent of the ripple amplitude. For these scenarios it was found that typically $17 \%$ of the NBI power is lost with a TF ripple of $\delta \sim 1.0 \%$. For standard JET operations with $\delta \sim 0.08 \%$ these losses are negligible.

A concern related to the particle losses is the power load on the JET in-vessel components when operating with an enhanced TF ripple. These loads were assessed prior to the experiments, again using the OFMC code mentioned above; yielding specific limits on the auxiliary heating power for a given value of the TF ripple amplitude and other plasma parameters. It was therefore important to select a plasma regime in which ITBs could be formed reliably at power levels within the limits of safe operations, but still with sufficient power margin to carry out experiments.

Two distinct plasma scenarios were used, as shown in figure 1. Both scenarios used a low triangularity plasma configuration ( $\delta^{\text {up }}=0.30, \delta^{\text {low }}=0.14, k=1.75$ ) and were heated early in the current ramp-up phase to optimize the current profile. In the first scenario, however, a lower hybrid current drive (LHCD) was applied before the main NBI and ICRH heating phase to create a negative, or reversed, magnetic shear (RS). In the second scenario the start 

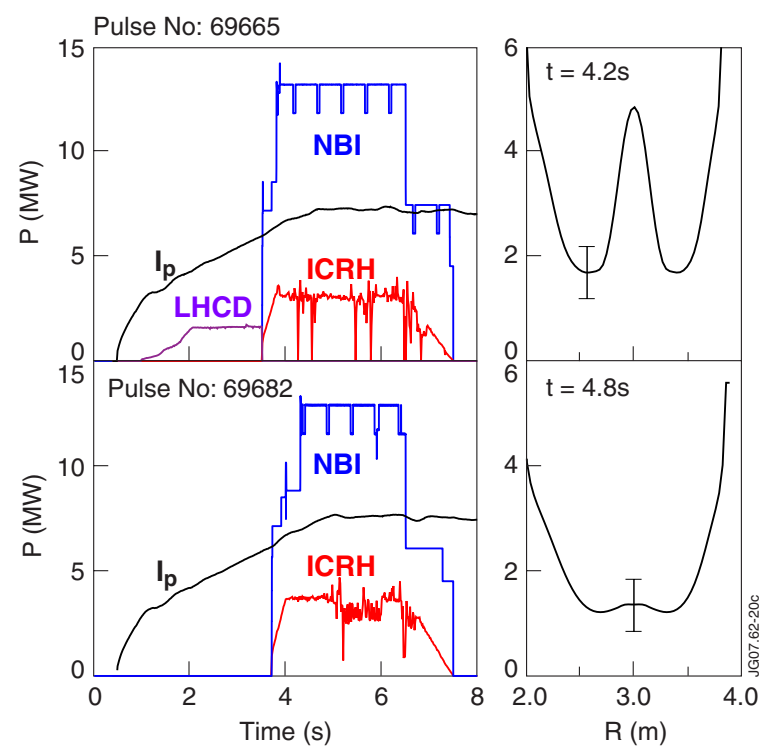

Figure 1. (Top) First scenario (RS) with an LHCD prelude $(t=1-3.5 \mathrm{~s})$ during the current rise phase and NBI (blue) and ICRH (red) switched on at $t=3.5 \mathrm{~s}$. The obtained $q$-profile in this is discharge shown on the right with a negative/reversed shear in the core and $q_{\min }$ at approximately $R=2.6$ and $3.5 \mathrm{~m}$. The value $q_{\text {min }}$ just below 2 at $t=4.2 \mathrm{~s}$ is also indicated by the observation of an Alfvén grand cascade at $t=3.9 \mathrm{~s}$ suggesting this coincides with $q_{\mathrm{min}}=2$. (Bottom) Second scenario (OS) with later NBI and ICRH heating $(t=3.8 \mathrm{~s})$. The $q$-profile is flat in the centre. The plasma current time traces [a.u.] are shown in black in the left graphs with the flat top starting at $t=5 \mathrm{~s}$. Both scenarios used $B_{\mathrm{t}}=2.2 \mathrm{~T}, I_{\mathrm{p}}=1.8 \mathrm{MA}$ and $q_{95}=4.1$. Both $q$-profiles are determined by the MSE diagnostic. Comparisons with other indicators of the $q$-profile, such as Alfvén cascades and other MHD events, suggest that the minimum $q$-value is often slightly higher than that measured by MSE at JET.

of the main heating was delayed and the LHCD omitted to create plasmas with a low positive, or optimized, magnetic shear (OS). This enabled a comparison to be made of ITB formation with different magnetic shear profiles. The average toroidal magnetic field, $B_{\mathrm{t}}=2.2 \mathrm{~T}$, and plasma current, $I_{\mathrm{p}}=1.8 \mathrm{MA}$ (giving $q_{95}=4.1$ ), allowed the TF ripple to be increased from the standard JET value of $\delta \sim 0.08 \%$ to $\delta \sim 1.0 \%$ while auxiliary heating by NBI and ICRH of $P_{\mathrm{NBI}}=17 \mathrm{MW}$ and $P_{\mathrm{ICRH}}=5 \mathrm{MW}$, respectively, was permitted throughout the ripple scan.

The TF ripple amplitude was increased from discharge to discharge. The amplitude was ramped during the first few seconds of the discharge and kept constant after $t=4 \mathrm{~s}$, i.e. during the main experimental phase. Furthermore, the power during the main heating phase was varied in several steps. This provided a series of discharges for each of the two ITB scenarios with various TF ripple amplitudes and absorbed powers. The effect of these variations on ITBs is discussed in the next section.

\section{The formation of ITBs}

The presence of an ITB is indicated by a local steepening of the temperature gradient. The temperature gradient can be expressed by a normalized value, $\rho_{T}^{*} \equiv \rho_{s} / L_{T}$, i.e. the ratio of the ion Larmor radius at the sound speed and the temperature gradient length [26]. In figure 2 the contours of the electron $\rho_{T}^{*}$ are shown as a function of time and the major radius. 


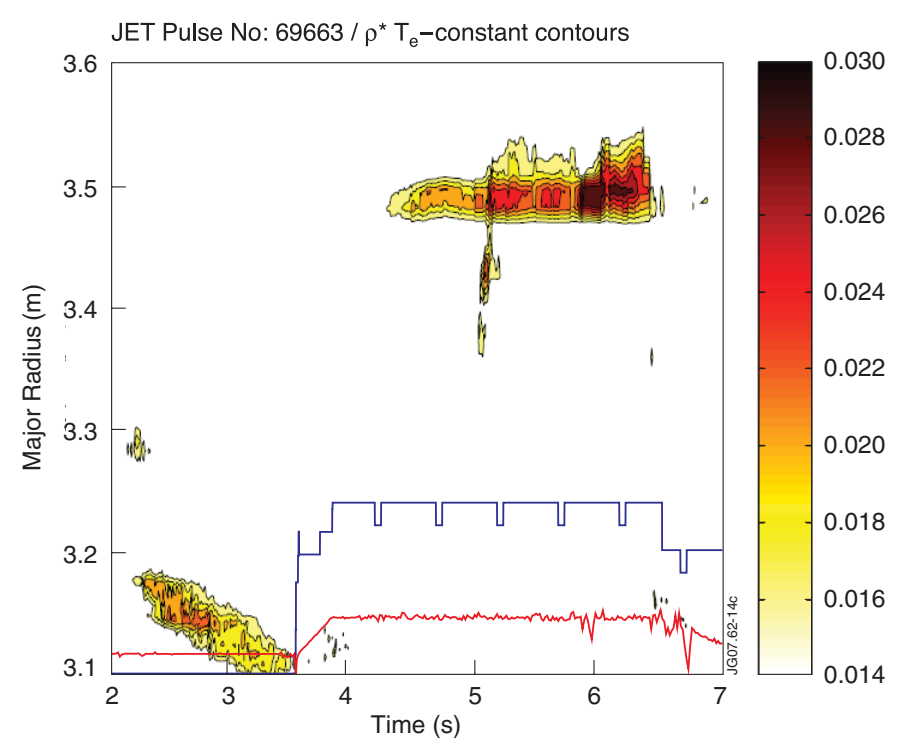

Figure 2. Contour plot of the $\rho_{T}^{*}$ values for a typical discharge using the scenario that produces a negative central shear (RS). The values are determined using the electron temperature profile and a minimum level of $\rho_{T}^{*}=0.014$ is shown. The time traces of auxiliary heating powers are shown in arbitrary units. During the prelude heating by LHCD $(t<3.6 \mathrm{~s})$ a barrier is seen close to the core of the plasma $\left(R_{\mathrm{O}}=3.0 \mathrm{~m}\right)$. A wider barrier is triggered during the main experimental phase at $t=4.4 \mathrm{~s}$, located at a major radius of $R=3.5 \mathrm{~m}(\rho \sim 0.6)$.

The minimum level shown in figure 2 is taken as $\rho_{T}^{*}=0.014$, which is an empirical threshold used to identify ITBs in JET [26]. The strength of the gradient in the electron temperature profile significantly exceeds this to reach a peak value of $\rho_{T}^{*}=0.031$ in this example. During the LHCD prelude $(t<3.6 \mathrm{~s})$ an ITB is seen close to the plasma centre $\left(R_{\mathrm{o}}=3.05 \mathrm{~m}\right)$. A wider barrier is triggered during the main heating phase at $t=4.25 \mathrm{~s}$, located at a major radius of $R=3.5 \mathrm{~m}$, or $\rho \sim 0.6$ ( $\rho$ is the normalized square-root poloidal flux coordinate). The ITB is always triggered close to the location of minimum $q$ (or zero magnetic shear) in this specific scenario (see figure 1) [13]. In these experiments, both the electron and ion temperature profiles showed increased gradients and the peak $\rho_{T}^{*}$ values were comparable.

It has been shown that the triggering of ITBs in plasmas with a reversed or negative shear is linked with the minimum $q$ reaching an integer value $[8,10,27]$. Similar observations were made in these experiments, which show the typical characteristics of grand cascades of Alfvén modes just prior to the appearance of the ITB. In figure 2 the ITB also forms at the location of the zero shear (and the minimum $q$ as shown in figure 1). The formation of the ITB in an optimized shear plasma has been shown to be related to MHD mode activity at the plasma edge $[8,10]$. In these ripple experiments the OS scenario showed a characteristic edge MHD coincident with the formation of ITBs at the outside edge of the region with flat $q$.

According to this criterion in $\rho_{T}^{*}$ for ITB identification, given above, the ITB in figure 2 was triggered at $t=4.25 \mathrm{~s}$, preceded by a grand cascade of Alfvén modes at $t=3.8 \mathrm{~s}$ (indicating that the $q$ minimum had an integer value at that time). In figure 3 the peak $\rho_{T}^{*}$ values obtained in these experiments are shown as a function of the total absorbed power. Note that the ITB strength does not necessarily depend on the absorbed power as the figures may suggest. It was found that the ITBs were triggered in almost all discharges, independent of the TF ripple amplitude, as seen in figure 3. There is one OS discharge in which $\rho_{T}^{*}$ did 

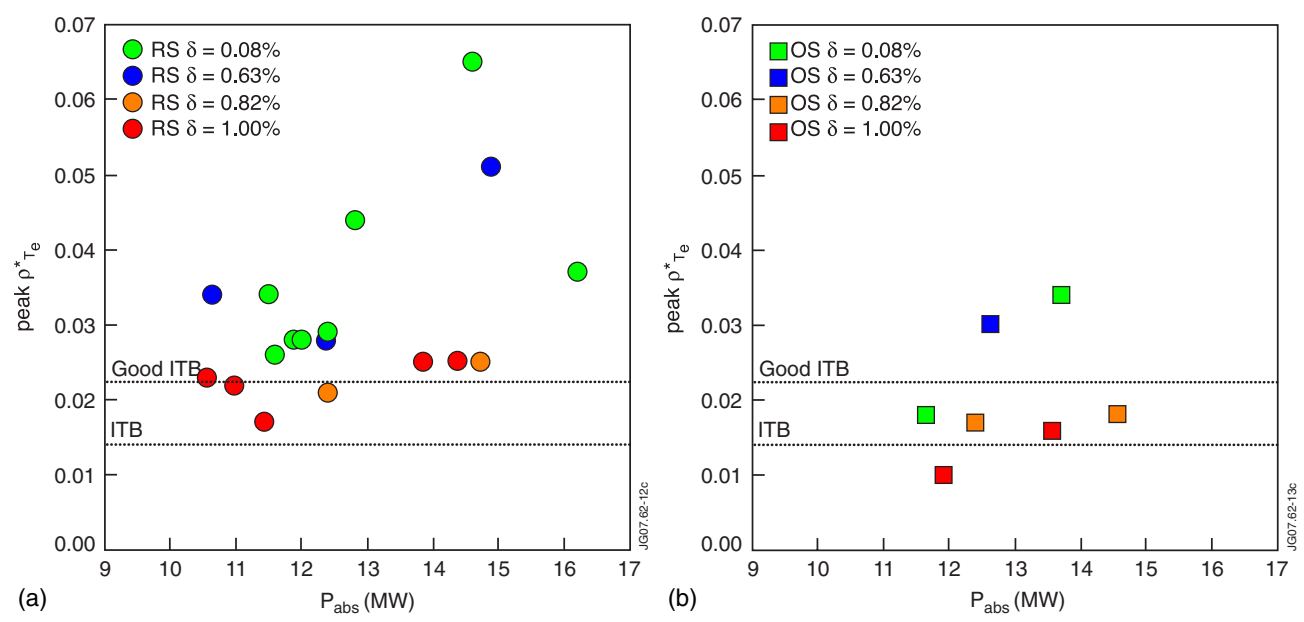

Figure 3. The maximum achieved $\rho_{T_{\mathrm{e}}}^{*}$ in each discharge as a function of the total absorbed power for the $(a) \mathrm{RS}$ and $(b)$ OS scenarios, respectively. The different colours indicate the TF ripple amplitude. The two levels of $\rho_{T_{\mathrm{e}}}^{*}>0.014$ (ITB) and $\rho_{T_{\mathrm{e}}}^{*}>0.023($ good ITB) indicate the trigger level and that when the ITB is significantly performance enhancing.

not reach 0.014 , possibly due to insufficient auxiliary heating power. Nevertheless, a local increase in the temperature gradient was seen in this plasma but the $\rho_{T}^{*}$ value remained below 0.01. Also the ITB location was not affected by the TF ripple amplitude. These observations suggest that in both scenarios, reversed and optimized shears, the characteristics of the current density, or $q$, profile were the dominant factors in the trigger mechanism for ITBs.

\section{The performance of ITBs}

Even after an ITB has been triggered it does not always 'grow' to cause a significant improvement in the performance of the tokamak discharge and sometimes the ITB criterion is only exceeded briefly. A well-developed ITB giving a significant plasma performance increase is defined here as having $\rho_{T}^{*}>0.023$. This level for a 'good' ITB is indicated by a horizontal line in figures $3(a)$ and $(b)$. Although ITBs were triggered in almost all discharges $\left(\rho_{T}^{*}>0.014\right)$, those with a larger TF ripple only had 'weak' ITBs. An increase in power in the OS scenario (figure 3(b)) did usually lead to stronger barriers, but even at higher levels of absorbed power the barriers remained weak for TF ripple amplitudes of $\delta \sim 0.8 \%$ to $\delta \sim 1.0 \%$. These weak ITBs were often short-lived, while those with steeper gradients could be maintained for the duration of the main heating phase (i.e. for a period of $0.5-1.5 \mathrm{~s}$ ).

The effect of the TF ripple on the ITB strength is more clearly visible in figure $4(a)$. Mainly weak $\left(\rho_{T}^{*}<0.023\right)$ ITBs are observed in discharges with a TF ripple of $\delta>0.8 \%$. A higher NBI particle loss fraction and lower absorbed power for larger TF ripple amplitude plasmas could not, however, explain the observed degradation of the ITB strength with the ripple amplitude. For example, in figure 4(a) RS discharges with different TF ripples but similar total absorbed power $\left(P_{\mathrm{abs}}=14.5 \pm 0.2 \mathrm{MW}\right)$ have been indicated. Even in this case discharges with a smaller TF ripple develop stronger transport barriers. The weaker ITBs observed at a high TF ripple have consequences for the overall performance of the plasma. In figure $4(b)$ the global plasma performance, expressed here as the normalized $\beta\left(\beta_{\mathrm{N}}\right)$, is shown to drop with the TF ripple amplitude for the four discharges discussed above. The presence 

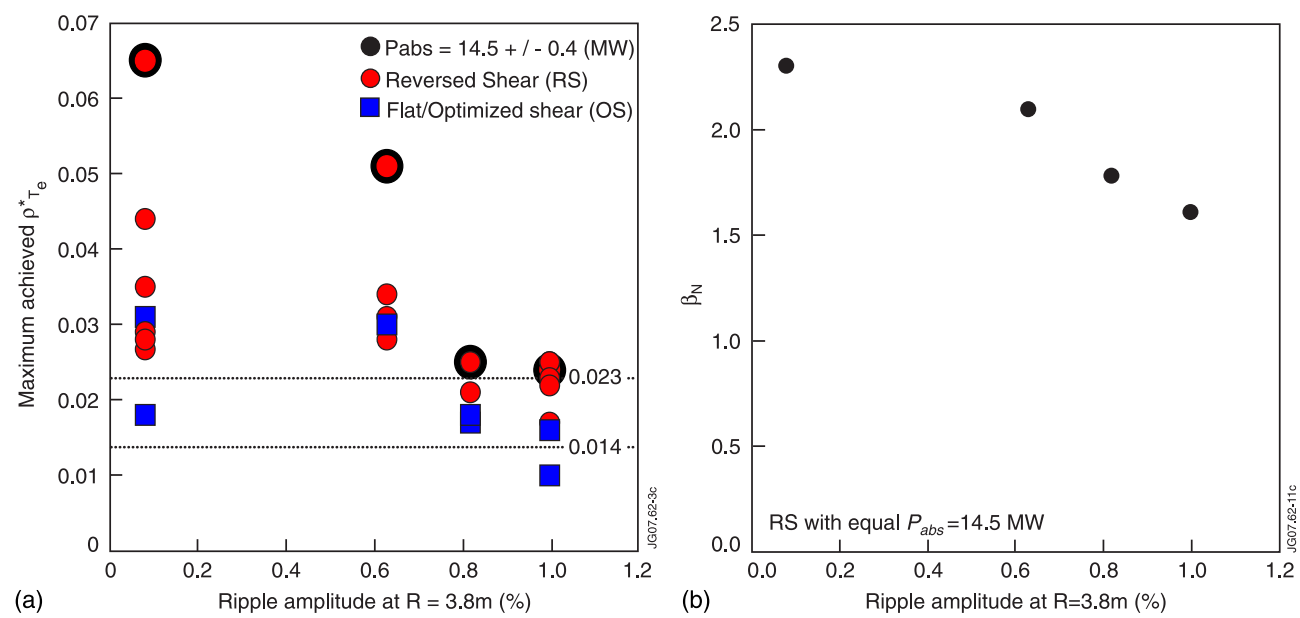

Figure 4. (a) The maximum achieved $\rho_{T_{e}}^{*}$ as a function of the TF ripple amplitude. The red circles represent the scenario with a reversed central shear (RS), while blue squares show the results with the optimized flat shear (OS). Four discharges (RS scenario) with equal absorbed power $P_{\mathrm{abs}}=14.5 \mathrm{MW}$ have been circled in black. $(b)$ The normalized $\beta\left(\beta_{\mathrm{N}}\right)$ for those four discharges shown circled $(a)$, taken at the time of maximum $\rho_{T_{\mathrm{e}}}^{*}$.
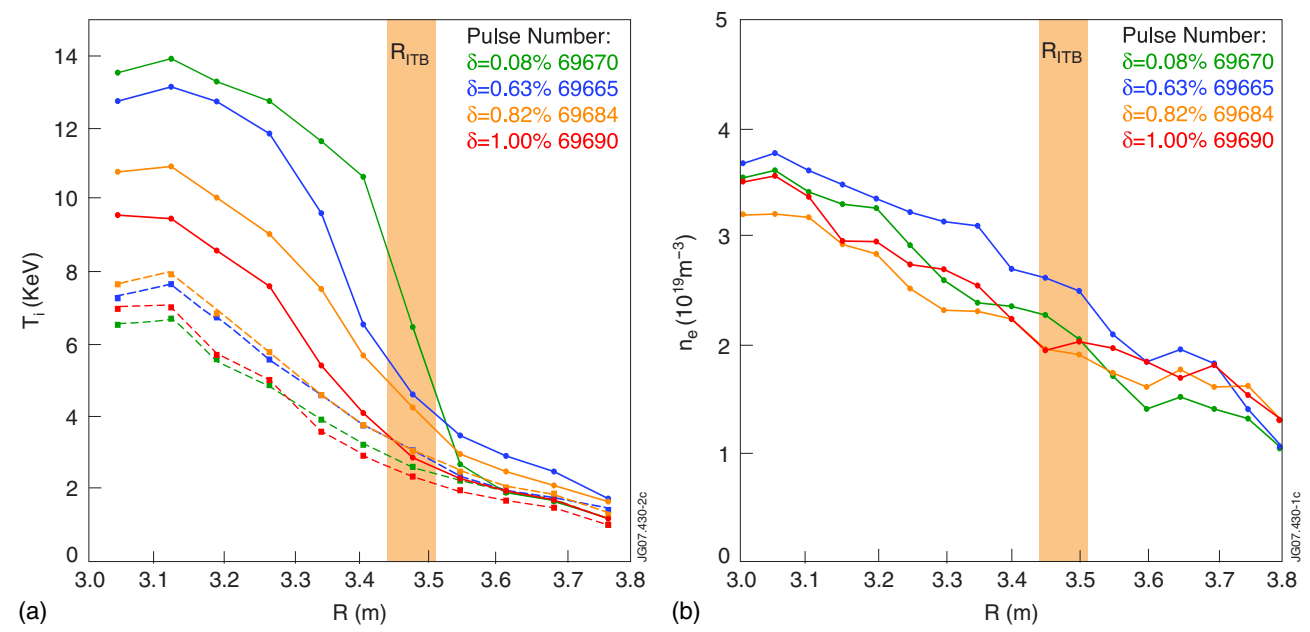

Figure 5. (a) The ion temperature profiles as measured by the CXRS diagnostic for the four discharges (RS scenario) with equal absorbed power $\left(P_{\mathrm{abs}}=14.5 \pm 0.2 \mathrm{MW}\right)$ taken at two times: at the time the ITB is triggered ( $\sim t=4.5 \mathrm{~s}$ ) (dashed lines) and the time of its maximum strength (solid lines). (b) The corresponding electron density profiles, as measured by Thomson scattering, at the time of the maximum strength of ITB. The centre of the plasma is at $R=3.05 \mathrm{~m}(\rho=0)$ and the separatrix at $R=3.85 \mathrm{~m}(\rho=1)$.

of a transport barrier generally improved the performance of this scenario above the baseline (without ITB) level of $\beta_{\mathrm{N}} \sim 1.4-1.5$, although the improvement was marginal at a TF ripple amplitude of $\delta=1.0 \%$. While at the lowest value of the TF ripple the ITB increased the plasma stored energy by almost $50 \%$. The difference in the ITB strength for these discharges is also evident from the ion temperature profiles shown in figure 5(a), with the weakest ITB when the TF ripple is the largest. In figure $5(b)$ the corresponding density profiles are shown. 

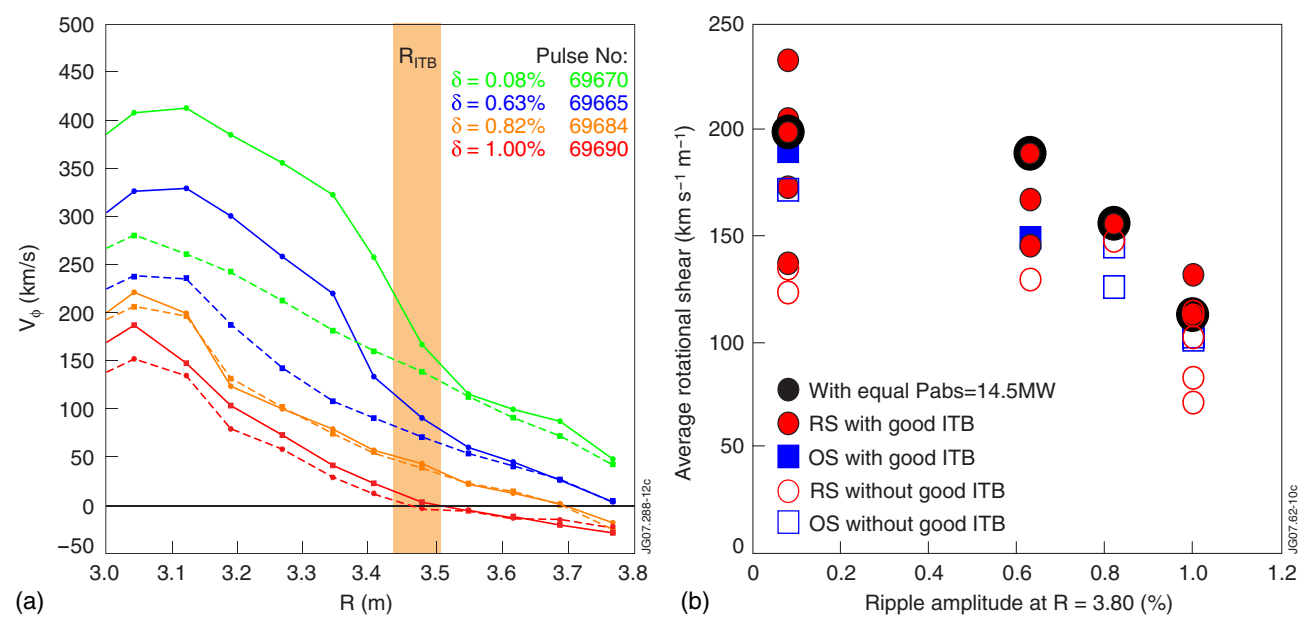

Figure 6. (a) The toroidal rotation profiles (for carbon) as a function of the normalized poloidal flux, $\rho$, for the four discharges (RS scenario) with equal absorbed power ( $P_{\mathrm{abs}}=14.5 \pm 0.2 \mathrm{MW}$ ) taken at two times: at the time the ITB is triggered $(\sim t=4.5 \mathrm{~s})$ (dashed lines) and at the time of its maximum strength (solid lines). The centre of the plasma is at $R=3.05 \mathrm{~m}(\rho=0)$ and the separatrix at $R=3.85 \mathrm{~m}(\rho=1)$. (b) The averaged gradient in the toroidal rotation profile at the foot of the ITB versus the TF ripple amplitude. The discharges with closed symbols did develop strong ITBs (i.e. $\left.\rho_{T}^{*}>0.023\right)$, while the open symbols indicate weak or no ITBs $\left(\rho_{T}^{*}<0.023\right)$. The four discharges in figures $4(b)$ and $5(a)$ are circled in black.

As mentioned in the introduction the TF ripple affects the plasma rotation [22]. Although the discharges shown in black circles in figure $4(b)$ may have had similar absorbed power, the total torque on these plasmas was reduced with the TF ripple amplitude. This resulted in significantly different toroidal rotation profiles as shown in figure 6(a). The plasma rotation has been measured by means of charge exchange recombination spectroscopy (CXRS). The measured quantities are those of carbon ions. For most JET plasmas the main ion species is found to be equal to the carbon rotation [28]. However, this assumption is not valid in the presence of strong ion temperature gradients. As shown in [28], discharges with temperature gradients such as those found in discharge \#69670 (shown in figure 5(a)) would result in a difference in carbon and deuterium rotation of $\Delta v / v_{\mathrm{C}} \sim 25-35 \%$ which is larger than the typical error bars on the measurements.

The toroidal rotation shown in figure $6(a)$ is lower for a higher TF ripple amplitude and even reverses direction in the outer part of the plasma. For $\delta=1 \%$ a region outside $R=3.48 \mathrm{~m}$ $(\rho=0.55)$ rotated in the counter-current direction (indicated as negative in the figure), while the JET NBI system injected momentum in the co-direction. For the two discharges that formed strong ITBs $(\delta<0.8 \%)$ the transport barrier causes a peaking of the core rotation. As described in [22] the observed counter rotation can be explained by TF ripple induced ion losses that cause a toroidal torque in the counter-current direction on the plasma. No measurable change was observed in the poloidal rotation at the time the ITBs were triggered, although a significant deviation from neo-classical poloidal rotation values had previously been observed during the subsequent evolution of similar ITBs at JET [29].

In this experiment ITBs were formed in the region of zero magnetic shear or at the outer edge of the low shear region in the RS and OS scenarios, respectively. These regions are at approximately $R \sim 3.4-3.5 \mathrm{~m}$, as seen in figure 1 . The location where the toroidal plasma rotation is zero moves well inside the plasma (up to $\rho \sim 0.6$ ) for $\delta>0.8 \%$ and almost reaches 
the outer edge of the ITB in these discharges. However, it is thought that the transport reduction responsible for the ITB is more likely to have been affected by the rotational shear than the absolute rotation. Figures $6(a)$ and $(b)$ show that the toroidal rotation gradient at the location of the ITBs $(R \sim 3.45-3.5 \mathrm{~m})$ reduced with the ripple amplitude. The gradients were determined just before the ITB was triggered and were averaged over the region $R=3.4-3.5 \mathrm{~m}$. Strong ITBs $\left(\rho_{T}^{*}>0.023\right)$ were only formed in discharges with a large toroidal torque and a large gradient in the toroidal rotation.

The rotational shear or shearing rate $\omega_{\boldsymbol{E} \times \boldsymbol{B}}$ has been determined for the four discharges shown in figure 6(a). The shearing rate can be expressed as

$$
\omega_{\boldsymbol{E} \times \boldsymbol{B}}=\frac{R B_{\theta}}{B} \frac{\partial}{\partial r}\left(\frac{E_{r}}{R B_{\theta}}\right)
$$

and the radial electric field, $E_{r}$, can be determined from the force balance equation:

$$
E_{r}=v_{\phi} B_{\theta}-v_{\theta} B_{\phi}+\frac{1}{Z n e} \frac{\partial P}{\partial r}
$$

The radial electric field depends on the toroidal $(\phi)$ and poloidal $(\theta)$ components of the velocity, $v$, and magnetic field, $B$, and on the gradient of the pressure, $P$ (for a species with an atomic charge $Z$ ).

Because a difference may exist between the rotation of the main ion species and that of carbon, measured carbon quantities have been used to determine the radial electric field. The poloidal rotation was assumed to be neo-classical and computed with the NCLASS code [30]. The assumption of neo-classical poloidal rotation, however, may be invalid when the local temperature gradient length approaches the ion Larmor radius. This condition may be reached in the presence of strong ITBs as discharge \#69670 shows in figure 5(a). Therefore, the shearing rate has been calculated at a time just prior to the ITB trigger, when the temperature gradients are still limited. The component of the shearing rate due to the gradient in the toroidal rotation is shown in figure $7(a)$ and the total $\boldsymbol{E} \times \boldsymbol{B}$ shearing rate in figure 7(b). Both were reduced as the TF ripple amplitude increased. From the comparison of figures $6(a)$ and $(b)$ it is clear that the toroidal rotation shear was the dominant component to the total $\boldsymbol{E} \times \boldsymbol{B}$ shear. These JET plasmas are in the low collisionality regime and it has been demonstrated that the last two terms in equation (2) tend to cancel out although the cancellation is not as strong for carbon as that for the deuterium species [9].

When the shearing rates are compared with the typical growth rate for ITG instabilities, $\gamma$, one finds that for the case with the smallest ripple $(\# 69670, \delta=0.08 \%) \omega_{\boldsymbol{E} \times \boldsymbol{B}} / \gamma \sim 0.8$ at the time when the ITB is triggered. Here the growth rate is calculated as discussed in [9]. As the gradients increase at the ITB this value may quickly rise above unity, mainly due to the increase in the gradient in toroidal rotation and possibly by a 'spin-up' of poloidal rotation [29]. However, the rotational shear for the discharge in figure 7 with a TF ripple of $\delta=1.00 \%$ (\#69690) is negligible and only a weak ITB is formed. The $\omega_{\boldsymbol{E} \times \boldsymbol{B}} / \gamma$ value, calculated using the neo-classical poloidal rotation, never exceeds $\sim 0.1$ in this pulse.

Similar calculations have been carried out for discharges with an optimized magnetic shear. In figure 3(b) two discharges with similar absorbed power of $P_{\text {abs }}=13.6 \pm 1 \mathrm{MW}$ are shown. The discharge with the highest TF ripple $(\delta=1.00 \%)$ and a low rotational shear did not form a strong ITB, while the one with a small TF ripple $(\delta=0.08 \%)$ and a larger rotational shear produced a barrier with $\rho_{T}^{*}>0.034$. 

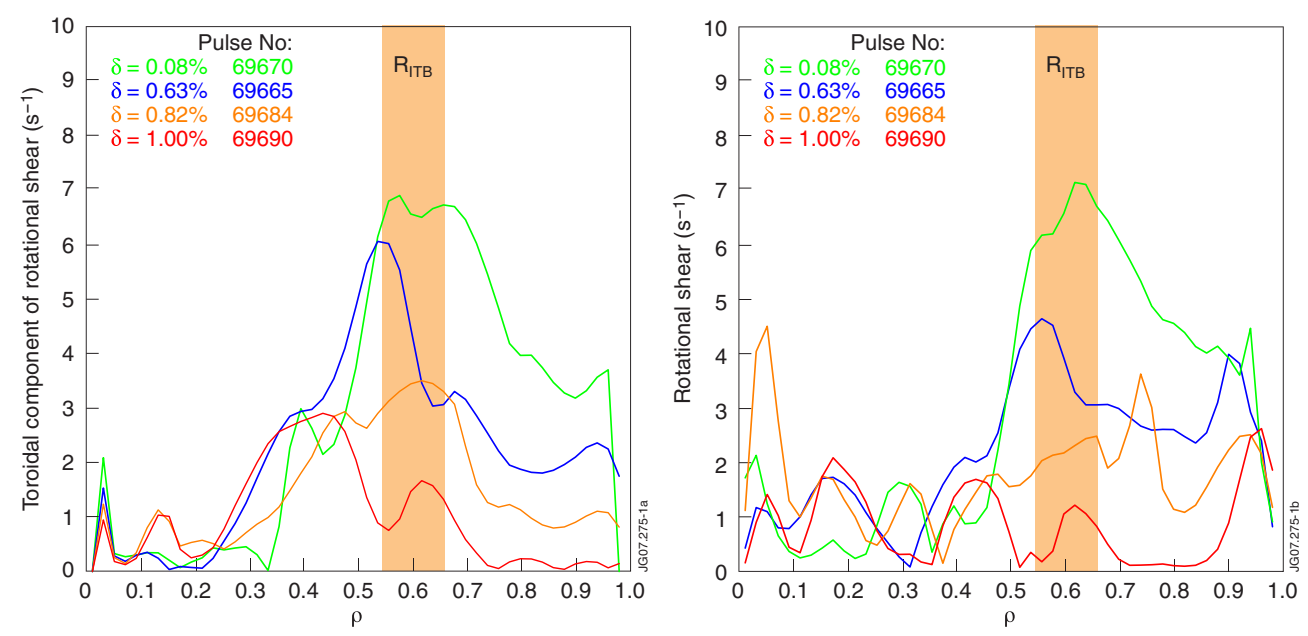

Figure 7. (a) The component of the rotational shear due to toroidal rotation as a function of the normalized poloidal flux, $\rho$, for the four discharges (RS scenario) with equal absorbed power ( $P_{\text {abs }}=14.5 \pm 0.2 \mathrm{MW}$ ). (b) The total $\boldsymbol{E} \times \boldsymbol{B}$ rotational shear profiles as for these four discharges assuming neo-classical poloidal rotation. Both figures were determined at the time the ITB is triggered $(\sim t=4.5 \mathrm{~s})$.

\section{Discussion}

The effects of an enhanced TF ripple on the formation and performance of ITBs have been discussed. The observations suggest that the ITBs are mainly affected indirectly due to the modification of the rotation profile by the field ripple. Besides the plasma rotation, ELMs can also affect the development of ITBs [24]. The influence of the TF ripple on the JET H-mode pedestal and ELMs is discussed in detail in [31]. In the experiments discussed in this paper the increased TF ripple amplitude led to irregular and smaller ELMs. The penetration of the pedestal crash into the plasma core was characterized by comparing the temperature profile before and after the ELM. For 'large' ELMs the profile is modified well inside the separatrix. At the standard ripple amplitude $(\delta \sim 0.08 \%)$ in these experiments the perturbations of a significant number of ELMs were able to affect the plasma at the location of the ITB while at larger ripple amplitudes $(\delta>0.5 \%)$ the number of such ELMs was significantly reduced. Nevertheless, this reduction in the ELM size did not seem to have a beneficial effect on the development of the ITB at a large ripple amplitude.

The JET TF ripple experiments have enabled the study of the formation of ITBs with different amounts of toroidal torque without altering other plasma parameters for ITB formation. For example, the relative fractions of NBI and ICRH power, fuelling and density were unchanged. Regardless of the TF ripple amplitude, ITBs were triggered in all RS discharges, suggesting that the trigger mechanism of this scenario may be insensitive to plasma rotation. Similar observations have been made in other experiments at JET [18]. The barrier formation in the reversed shear scenario is often associated with the minimum $q$ reaching an integer value [12]. More power is required to form ITBs in the optimized shear scenarios where this particular trigger mechanism is generally absent. A larger fraction of the NBI power was lost in discharges with a high TF ripple; hence a larger input power was required to achieve ITBs. Nevertheless, comparing discharges with similar total absorbed power, it was again found that those with a larger TF ripple and lower toroidal rotation shear formed only weak ITBs. 
These experiments have shown that, although the ITB trigger was unaffected, the subsequent development of ITBs to produce steep temperature gradients in JET was degraded as the TF ripple increased. Increasing the TF ripple reduced the toroidal rotation and modified the toroidal rotation profile. Extrapolating the TF ripple effects that are found on JET to other devices requires care. The mechanism by which the TF ripple affects plasma rotation and rotational shear depends on the details of the applied torque (e.g. from an NBI system, fast particle losses), which can differ significantly from those at JET [22]. For example, in JT-60U significant plasma rotation in the counter-current direction, and thus rotational shear, was observed with near perpendicular NBI in the presence of a TF ripple of $\delta \sim 1 \%$ [32].

The results presented in this paper on OS and RS ITB scenarios suggest that increased rotational shear can lead to the formation of stronger ITBs. After the initial trigger, which appears to be related mainly to the characteristics of the $q$-profile, the rotational shear seems to play a key role in further suppression of turbulence and strengthening of the transport barrier. This in turn enhances the rotational shear, providing a positive feedback. The ITB trigger and the feedback mechanism responsible for the further development of the barrier would, therefore, appear to be governed by different physical processes.

\section{Acknowledgments}

The authors would like to thank JAEA for the use of the OFMC code for the studies presented in this paper. This research was funded partly by the United Kingdom Engineering and Physical Sciences Research Council and by the European Communities under the contract of Association between EURATOM and UKAEA. The views and opinions expressed herein do not necessarily reflect those of the European Commission. This work was carried out within the framework of the European Fusion Development Agreement.

(c) Euratom 2008.

\section{References}

[1] Kikuchi M 1990 Nucl. Fusion 30265

[2] Luce T C et al 2003 Nucl. Fusion 43321

[3] Litaudon X et al 2002 Plasma Phys. Control. Fusion 441057

[4] Gormezano C et al 2007 Nucl. Fusion 47 S285

[5] Green B J et al 2003 Plasma Phys. Control. Fusion 45687

[6] Connor J W et al 2004 Nucl. Fusion 44 R1

[7] Challis C D et al 2004 Plasma Phys. Control. Fusion 46 B23

[8] Challis C D et al 2001 Plasma Phys. Control. Fusion 43861

[9] Tala T et al 2001 Plasma Phys. Control. Fusion 43507

[10] Synakowski E J 1998 Plasma Phys. Control. Fusion 40581

[11] Shirai H et al 2000 Plasma Phys. Control. Fusion 42 A109

[12] Joffrin E et al 2002 Plasma Phys. Control. Fusion 441739

[13] Joffrin E et al 2003 Nucl. Fusion 431167

[14] Fujita T et al 2001Phys. Rev. Lett. 87245001

[15] Hawkes N C et al 2001 Phys. Rev. Lett. 87115001

[16] Hawkes N C et al 2002 Plasma Phys. Control. Fusion 441105

[17] Challis C D et al 2002 Plasma Phys. Control. Fusion 441031

[18] Hawkes N C et al 2007 Ion transport barrier formation with low E $\times$ B shear in JET Proc. 34th EPS Conf. on Plasma Phys. Control. Fusion (Warsaw, 2007)

[19] Synakowski E J et al 1997Phys. Rev. Lett. 782972

[20] Sakamoto Y et al 2001 Nucl. Fusion 41865

[21] Tubbing B J D and the JET team 1995 Proc. 22nd EPS Conf. on Plasma Physics and Control. Fusion (Bournemouth, 1995) vol 19C pIV-001 
[22] de Vries P C et al 2008 Nucl. Fusion 48035007

[23] 2007 ITER Project Integration Document ITER_D_2234RH ed J How (Version 3 2007)

[24] Sarazin Y et al 2002 Plasma Phys. Control. Fusion 442445

[25] Shinohara K et al 2003 Nucl. Fusion 43586

[26] Tresset G et al 2002 Nucl. Fusion 42520

[27] Sharapov S E et al 2001Phys. Lett. A 289127

[28] Testa D et al 2002 Phys. Plasmas 9243

[29] Crombé K et al 2005 Phys. Rev. Lett. 95155003

[30] Houlberg W et al 1997 Phys. Plasmas 43231

[31] Saibene G et al 2007 Toroidal field ripple effects on H-modes in JET and implications for ITER' Proc. 34th EPS Conf. on Plasma Phys. Control. Fusion (Warsaw, 2007)

[32] Koide Y et al 1993 Plasma Phys. Control. Nucl. Fusion Res. 1777 